\title{
A NEW DERIVATION OF THE BAYESIAN BOUNDS FOR PARAMETER ESTIMATION
}

\author{
Alexandre Renaux ${ }^{(1)}$, Philippe Forster ${ }^{(2)}$ and Pascal Larzabal ${ }^{(1)}$ \\ (1) SATIE / UMR 8029, ENS Cachan 61, avenue du Président Wilson 94235 Cachan Cedex, France. \\ (2) GEA, IUT Ville d'Avray, 1 Chemin Desvallières, 92410 Ville d'Avray, France.
}

\begin{abstract}
This paper deals with minimal bounds in the Bayesian context. We express the minimum mean square error of the conditional mean estimator as the solution of a continuum constrained optimization problem. And, by relaxing these constraints, we obtain the bounds of the Weiss-Weinstein family. Moreover, this method enables us to derive new bounds as the Bayesian version of the deterministic Abel bound.
\end{abstract}

\section{INTRODUCTION}

We study the problem of establishing minimal bounds on the Mean Square Error (MSE) in the case of a random parameter modelling, extending in this way previous results on deterministic bounds [1] [2]. In the Bayesian theory, it is well known that the conditional mean estimator (MMSEE) gives the minimum MSE and consequently, the best attainable Bayesian lower bound. Nevertheless, obtention of a MMSEE MSE closed form expression is often difficult and, most of the time, impossible. Then, in order to know the ultimate performances of an estimator, it is important to have a lower bound on the MSE. Of course, this bound is expected to be tight even when the scenario is critical (small Signal to Noise Ratio(SNR) and/or small number of snapshots). Let us note that this is not the case for the Bayesian Cramér-Rao bound which is usually used in the Bayesian framework [3]. To compense for this lack, and despite their complexity, two families of Bayesian bounds are particularly appreciated for their tightness (see recent papers [4][5]):

- The Ziv-Zakai family which derives from an hypothesis test [6].

- The Weiss-Weinstein family which derives from a covariance inequality principle [7].

These approaches have provided a plethora bounds: ZivZakai [6], Bellini-Tartara [8], Chazan-Zakai-Ziv [9], Weinstein [10],... for the Ziv-Zakai family and Bayesian CramérRao [3], Bayesian Bhattacharyya [3], Bobrovsky-Zakai [11],

This work was partially supported by the European Community Contract no. 507325, NEWCOM
Weiss-Weinstein [7]... for the Weiss-Weinstein family. As a consequence, it can be difficult within each family to see the link between these bounds. Therefore, a unification work seems important to understand the various underlaying hypothesis for bounds establishment. This has been done for the Ziv-Zakai family by Kristine Bell [12].

For the Weiss-Weinstein family, a first approach has been undertaken by Weiss and Weinstein [13]. But, this approach seems difficult to exploit since the authors introduce an arbitrary function for which they do not give any rule for deriving.

The purpose of this article is to present a new unified approach for the establishment of Bayesian bounds as the solution of a constrained optimization problem. Our approach consists in expressing the MSE of the MMSEE (best Bayesian bound) as the result of an original constrained optimization problem. Next, by relaxing these constraints, we naturally obtain a method to establish Bayesian bounds. We show that this new concept provides all the classical Bayesian bounds of the Weiss-Weinstein family, and moreover it allows us to derive new bounds.

\section{A NEW FORMULATION OF THE MINIMUM MSE}

\subsection{Constrained optimization problem}

Let $\mathbf{x} \in \Omega$ be an observation vector and $\theta \in \Theta$ be a scalar parameter that we want to estimate. Let $f(\mathbf{x}, \theta)$ and $g(\mathbf{x}, \theta)$ two functions of $I R^{N} \rightarrow I R$. Let

$$
\langle f(\mathbf{x}, \theta), g(\mathbf{x}, \theta)\rangle=\iint_{\Omega} f(\mathbf{x}, \theta) g(\mathbf{x}, \theta) d \theta d \mathbf{x},
$$

a scalar product of these two functions, and its associate norm $\|f(\mathbf{x}, \theta)\|^{2}=\langle f(\mathbf{x}, \theta), f(\mathbf{x}, \theta)\rangle$.

Theorem 1: Let $u(\mathbf{x}, \theta), g_{0}(\mathbf{x}, \theta), \ldots, g_{K}(\mathbf{x}, \theta)$, a set of functions of $I R^{N} \rightarrow I R$ and $c_{0}, c_{1}, \ldots, c_{K}, K+1$ real numbers. Recall the result yet used in [1]: the minimum value of $\|u(\mathbf{x}, \theta)\|^{2}$ with the $K+1$ constraints

$$
\left\langle u(\mathbf{x}, \theta), g_{i}(\mathbf{x}, \theta)\right\rangle=c_{i} \text { for } i=0, \ldots, K
$$


is given by

$$
\min \|u(\mathbf{x}, \theta)\|^{2}=\mathbf{c}^{\mathrm{T}} \mathbf{G}^{-1} \mathbf{c}
$$

with

$$
\mathbf{c}=\left[c_{0}, c_{1}, \ldots, c_{K}\right]^{\mathrm{T}},
$$

and

$$
G_{m, n}=\left\langle g_{m}(\mathbf{x}, \theta), g_{n}(\mathbf{x}, \theta)\right\rangle .
$$

\subsection{New formulation of the minimal MSE}

The MSE of the MMSEE is the solution of the following constrained optimization problem

$$
\left\{\begin{array}{c}
\min \iint_{\Omega} v^{2}(\mathbf{x}, \theta) p(\mathbf{x}, \theta) d \theta d \mathbf{x} \\
\text { subject to } v(\mathbf{x}, \theta)=\hat{\theta}(\mathbf{x})-\theta
\end{array}\right.
$$

On the other hand, with $v(\mathbf{x}, \theta)=\frac{u(\mathbf{x}, \theta)}{\sqrt{p(\mathbf{x}, \theta)}}$, theorem 1 gives the solution of the following problem for $i=0, \ldots, K$.

$$
\left\{\begin{array}{l}
\min \iint_{\Omega} v^{2}(\mathbf{x}, \theta) p(\mathbf{x}, \theta) d \theta d \mathbf{x} \\
\text { subject to } \iint_{\Omega} v(\mathbf{x}, \theta) g_{i}(\mathbf{x}, \theta) \sqrt{p(\mathbf{x}, \theta)} d \theta d \mathbf{x}=c_{i}
\end{array}\right.
$$

First, we will show that the constraint of the optimization problem (6) is equivalent to a continuum of appropriate constraints of the optimization problem (7). This will permit us to establish Bayesian bounds with theorem 1 .

Noting $f(\mathbf{x}, \theta)$ a function such that

$$
\iint_{\Omega} f(\mathbf{x}, \theta) d \theta d \mathbf{x}=1
$$

let us introduce the four following assumptions A1, A2, A3, A4 on $v(\mathbf{x}, \theta)$

$A l: \forall f(\mathbf{x}, \theta)$

$$
\iint_{\Omega} v(\mathbf{x}, \theta) \frac{\partial f(\mathbf{x}, \theta)}{\partial \theta} d \theta d \mathbf{x}=1 .
$$

$A 2: \forall f(\mathbf{x}, \theta)$ and $\forall h$

$$
\iint_{\Omega} v(\mathbf{x}, \theta)(f(\mathbf{x}, \theta+h)-f(\mathbf{x}, \theta)) d \theta d \mathbf{x}=h .
$$

$A 3: \forall f(\mathbf{x}, \theta), \forall h$ and $\forall s$

$$
\iint_{\Omega} v(\mathbf{x}, \theta) w(\mathbf{x}, \theta, h, s) f(\mathbf{x}, \theta) d \theta d \mathbf{x}
$$

$$
=h \iint_{\Omega}\left(\frac{f(\mathbf{x}, \theta-h)}{f(\mathbf{x}, \theta)}\right)^{1-s} f(\mathbf{x}, \theta) d \theta d \mathbf{x}
$$

with $w(\mathbf{x}, \theta, h)=\left(\frac{f(\mathbf{x}, \theta+h)}{f(\mathbf{x}, \theta)}\right)^{s}-\left(\frac{f(\mathbf{x}, \theta-h)}{f(\mathbf{x}, \theta)}\right)^{1-s}$.

A4:

$$
v(\mathbf{x}, \theta)=q(\mathbf{x})-\theta,
$$

where $q(\mathbf{x})$ is function of $\mathbf{x}$ only.

The three following theorems (the proofs are given in the appendix) will permit us to establish Bayesian bounds in the next section.

Theorem 2:

$$
A 1 \Leftrightarrow A 4
$$

Theorem 3:

$$
A 2 \Leftrightarrow A 4
$$

Theorem 4:

$$
A 3 \Leftrightarrow A 4
$$

Theorems 2, 3, and 4 establish the equivalence between (6) and (7) with an appropriate continuum of constraints. In the next section we will relax this continuum to a discrete set of constraints $(K<\infty)$ for which $f(\mathbf{x}, \theta)=p(\mathbf{x}, \theta)$ (the joint pdf of the problem) and for a finite choice of parameters $h$ and $s$. By relaxation of these constraints, the solution of the optimization problem (7) will give weaker bounds in comparison with the MSE of the MMSEE. Moreover, since the best bound is given by the MSE of the MMSEE (without joint bias) it seems natural to introduce the following constraint $\iint_{\Omega} v(\mathbf{x}, \theta) p(\mathbf{x}, \theta) d \theta d x=0$.

\section{APPLICATION TO ESTABLISHMENT OF BAYESIAN BOUNDS}

In the following, we put $f(\mathbf{x}, \theta)=p(\mathbf{x}, \theta)$ the joint pdf of the problem.

\subsection{Bayesian Cramér-Rao and Bhattacharyya bounds}

By successive derivation of the equality used in A2 with respect to $h$ in $h=0$ we obtain $\int_{\Omega} \int_{\Theta} v(\mathbf{x}, \theta) \frac{\partial^{i} f(\mathbf{x}, \theta)}{\partial \theta^{i}} d \theta d \mathbf{x}=\delta_{1 i}$ where $\delta_{1 i}=\left\{\begin{array}{l}1 \text { for } i=1 \\ 0 \text { for } i>1\end{array}\right.$. It follows the set of $K+1$ constraints

$$
\mathbf{g}=\frac{1}{\sqrt{p(\mathbf{x}, \theta)}}\left[\begin{array}{c}
p(\mathbf{x}, \theta) \\
\frac{\partial p(\mathbf{x}, \theta)}{\partial \theta} \\
\vdots \\
\frac{\partial^{K} p(\mathbf{x}, \theta)}{\partial \theta^{K}}
\end{array}\right]
$$


and

$$
\mathbf{c}=[0,1,0, \cdots, 0]^{\mathrm{T}} .
$$

Theorem 1 gives

$$
\begin{aligned}
M S E_{\min } & =\mathbf{c}^{\mathrm{T}} \mathbf{G}^{-1} \mathbf{c} \\
& =[1,0, \cdots, 0] \mathbf{B}^{-1}[1,0, \cdots, 0]^{\mathrm{T}} \\
& =\left(\mathbf{B}^{-1}\right)_{1,1},
\end{aligned}
$$

where for $i, j \geq 1$

$$
B_{i, j}=\int_{\Omega} \int_{\Theta} \frac{1}{p(\mathbf{x}, \theta)} \frac{\partial^{i} p(\mathbf{x}, \theta)}{\partial \theta^{i}} \frac{\partial^{j} p(\mathbf{x}, \theta)}{\partial \theta^{j}} d \theta d \mathbf{x} .
$$

This is the Bayesian Bhattacharyya bound [3]. And, in the particular case where $K=1$,

$$
M S E_{\min }=\left(\int_{\Omega} \int_{\Theta} \frac{1}{p(\mathbf{x}, \theta)} \frac{\partial p(\mathbf{x}, \theta)}{\partial \theta} d \theta d \mathbf{x}\right)^{-1},
$$

which is the Bayesian Cramér-Rao bound [3].

\subsection{Reuven-Messer bound and Bobrovsky-Zakai bound}

By sampling the range of parameter $h$ of the equality used in $\mathrm{A} 2$, the set of $K+1$ constraints is obtained

$$
\mathbf{g}=\frac{1}{\sqrt{p(\mathbf{x}, \theta)}}\left[\begin{array}{c}
p(\mathbf{x}, \theta) \\
p\left(\mathbf{x}, \theta+h_{1}\right) \\
\vdots \\
p\left(\mathbf{x}, \theta+h_{K}\right)
\end{array}\right]
$$

and

$$
\begin{aligned}
\mathbf{c} & =\left[0, h_{1}, h_{2}, \cdots, h_{K}\right]^{\mathrm{T}} \\
& =\left[0, \mathbf{h}^{\mathrm{T}}\right]^{\mathrm{T}},
\end{aligned}
$$

with $h_{i} \in I R$ for $i=1, \ldots, K$ and $\mathbf{h}=\left[h_{1}, h_{2}, \cdots, h_{K}\right]^{\mathrm{T}}$.

Theorem 1 gives

$$
\begin{aligned}
M S E_{\min } & =\mathbf{c}^{\mathrm{T}} \mathbf{G}^{-1} \mathbf{c} \\
& =\mathbf{h}^{\mathrm{T}}\left(\mathbf{D}-\mathbf{1 1}^{\mathrm{T}}\right)^{-1} \mathbf{h},
\end{aligned}
$$

where for $i, j \geq 1$

$$
D_{i, j}=\iint_{\Omega} \frac{p\left(\mathbf{x}, \theta+h_{i}\right) p\left(\mathbf{x}, \theta+h_{j}\right)}{p(\mathbf{x}, \theta)} d \theta d \mathbf{x} .
$$

Since the parameters $h_{i} i=1 \cdots K$ are free, by maximizing over $h_{i}$ one obtains a particular case of the ReuvenMesser hybrid bound (all parameters random) [14]. This is given by

$$
M S E_{\min }=\lim _{K \rightarrow \infty} \max _{\mathbf{h}} \mathbf{h}^{\mathrm{T}}\left(\mathbf{D}-\mathbf{1 1}^{\mathrm{T}}\right)^{-1} \mathbf{h} .
$$

In the particular case where $K=1$, one obtains

$$
M S E_{\min }=\max _{h} \frac{h^{2}}{\iint_{\Omega} \frac{p^{2}(\mathbf{x}, \theta+h)}{p(\mathbf{x}, \theta)} d \theta d \mathbf{x}-1},
$$

which is the Bobrovsky-Zakai bound (another particular case of the hybrid Reuven-Messer bound) [11].

\subsection{Weiss-Weinstein bound}

Finally, by sampling the range of parameters $h$ and $s$ of the equality used in $\mathrm{A} 3$, the set of $K+1$ constraints is obtained

$$
\left[\begin{array}{c}
\mathbf{g}=\sqrt{p(\mathbf{x}, \theta) \times} \\
1 \\
L^{s_{1}}\left(\mathbf{x} \mid \theta+h_{1}, \theta\right)-L^{1-s_{1}}\left(\mathbf{x} \mid \theta-h_{1}, \theta\right) \\
L^{s_{2}}\left(\mathbf{x} \mid \theta+h_{2}, \theta\right)-L^{1-s_{2}}\left(\mathbf{x} \mid \theta-h_{2}, \theta\right) \\
\vdots \\
L^{s_{K}}\left(\mathbf{x} \mid \theta+h_{K}, \theta\right)-L^{1-s_{K}}\left(\mathbf{x} \mid \theta-h_{K}, \theta\right)
\end{array}\right]
$$

and

$$
\mathbf{c}=\left[\begin{array}{c}
0 \\
h_{1} E\left[L^{1-s_{1}}\left(\mathbf{x} \mid \theta-h_{1}, \theta\right)\right] \\
h_{2} E\left[L^{1-s_{2}}\left(\mathbf{x} \mid \theta-h_{2}, \theta\right)\right] \\
\vdots \\
h_{K} E\left[L^{1-s_{K}}\left(\mathbf{x} \mid \theta-h_{K}, \theta\right)\right]
\end{array}\right]=\left[0, \boldsymbol{\xi}^{\mathrm{T}}\right]^{\mathrm{T}},
$$

with $L\left(\mathbf{x} \mid \theta_{1}, \theta_{2}\right) \triangleq \frac{p\left(\mathbf{x}, \theta_{1}\right)}{p\left(\mathbf{x}, \theta_{2}\right)}$.

Theorem 1 gives

$$
M S E_{\min }=\mathbf{c}^{\mathrm{T}} \mathbf{G}^{-1} \mathbf{c}=\boldsymbol{\xi}^{\mathrm{T}} \mathbf{W}^{-1} \boldsymbol{\xi}
$$

and

$$
\begin{gathered}
W_{i, j}= \\
E\left[\left(L^{s_{i}}\left(\mathbf{x} \mid \theta+h_{i}, \theta\right)-L^{1-s_{i}}\left(\mathbf{x} \mid \theta-h_{i}, \theta\right)\right)\right. \\
\left.\times\left(L^{s_{j}}\left(\mathbf{x} \mid \theta+h_{j}, \theta\right)-L^{1-s_{j}}\left(\mathbf{x} \mid \theta-h_{j}, \theta\right)\right)\right],
\end{gathered}
$$

for $i, j \geq 1$.

Since the parameters $h_{i}$, and $s_{i}(i=1 \cdots K)$ are free, by maximizing over $h_{i}$, and $s_{i}$, we obtain the Weiss and Weinstein bound [7]

$$
M S E_{\min }=\lim _{K \rightarrow \infty} \max _{h_{1}, h_{2}, \cdots, h_{K} \sin _{1}, s_{2}, \cdots, s_{K}} \boldsymbol{\xi}^{\mathrm{T}} \mathbf{W}^{-1} \boldsymbol{\xi} .
$$

\subsection{Application for new bounds establishment: one ex- ample}

The proposed approach is interesting because it can be used to create new bounds with an appropriate subset of constraints. Here, our main motivation is to establish a bound which will be close to the MMSEE MSE, with a complexity 
slightly upper than the Bobrovsky-Zakai bound. Indeed, by combining a constraint of (9) and a constraint of (10), i.e.

$$
\mathbf{g}=\frac{1}{\sqrt{p(\mathbf{x}, \theta)}}\left[p(\mathbf{x}, \theta), \frac{\partial p(\mathbf{x}, \theta)}{\partial \theta}, p(\mathbf{x}, \theta+h)\right]^{\mathrm{T}},
$$

and

$$
\mathbf{c}=[0,1, h]^{\mathrm{T}} .
$$

One obtains straightforwardly, after inverting $\mathbf{G}(3 \times 3$ matrix) and maximization over $h$

$$
M S E_{\min } \geq \max _{h} \frac{B C R B^{-1}+B Z B^{-1}-2 \phi}{B C R B^{-1} B Z B^{-1}-\phi^{2}} .
$$

Where $B C R B$ is the Bayesian Cramér-Rao bound, $B Z B$ is the Bobrovsky-Zakai bound without maximization over $h$, and

$$
\phi=\frac{1}{h} \iint_{\Omega} \frac{\partial \ln p(\mathbf{x}, \theta)}{\partial \theta} p(\mathbf{x}, \theta+h) d \theta d \mathbf{x} .
$$

Note that our bound can be seen as the Bayesian version of the deterministic Abel bound [15], at the first order.

\section{COMPARISON OF THE PROPOSED BOUND WITH THE BCRB AND THE BZB IN A SPECTRAL ANALYSIS CONTEXT}

\subsection{Bounds derivations}

It is a well known result that for critical scenarios, i.e. when the number of observation and/or the SNR are small, the Bayesian Cramér-Rao Bound is too optimistic. Particularly, it does not predict the threshold effect of estimators. Then, bounds tighter than the Bayesian Cramér-Rao Bound are beneficial. Following this idea, our previous Bayesian Abel bound is derived and analyzed in a classical spectral analysis context.

The model is the following

$$
\mathbf{x}=\rho \mathbf{s}+\mathbf{n}
$$

where $\mathbf{x}$ is the observation vector, $\rho^{2}$ is the SNR,

$$
\mathbf{s}=\left[1, e^{j \theta}, \ldots, e^{j(N-1) \theta}\right]^{\mathrm{T}},
$$

is the signal, $\mathbf{n}$ is the noise vector which is assumed circular, complex Gaussian with zero mean and covariance matrix $\mathbf{I}_{N}$, and $\theta$ is the parameter of interest (the pulsation in this problem). The a priori pdf of $\theta$ is taken here Gaussian with zero mean and variance $\sigma_{\theta}^{2}$.

Then, we have straightforwardly

$$
p(\mathbf{x} \mid \theta)=\frac{1}{\pi^{N}} e^{-\|\mathbf{x}-\rho \mathbf{s}\|^{2}},
$$

and

$$
p(\theta)=\frac{1}{\sqrt{2 \pi} \sigma_{\theta}^{2}} e^{-\frac{\theta^{2}}{2 \sigma_{\theta}^{2}}} .
$$

By using eqn. (34) and (35) in eqn. (16), (22), and with some algebraic effort not reported here due to the lack of space, we obtain the following closed forms

$$
\begin{aligned}
B C R B & =\frac{3 \sigma_{\theta}^{2}}{\sigma_{\theta}^{2} \rho^{2} N(2 N-1)(N-1)+3}, \\
B Z B & =\max _{h} \frac{h^{2}}{e^{4 \rho^{2} \Sigma_{1}+\frac{h^{2}}{\sigma_{\theta}^{2}}}-1},
\end{aligned}
$$

where $\Sigma_{1}$ is given by

$$
\begin{gathered}
\Sigma_{1}=N+\frac{1}{2}[\cos (N h) \\
\left.-\frac{\sin (N h)}{\sin (h)}(\cos (h)+1)-1\right]
\end{gathered}
$$

The proposed bound is given by eqn. (30), which depends on the $B C R B$ and $B Z B$ (without maximization over $h$ ) given above and of $\phi$ which is given in our case by

$$
\phi=\frac{2 \rho^{2}}{h} \Sigma_{2}+\frac{1}{\sigma_{\theta}^{2}},
$$

where

$$
\Sigma_{2}=\frac{1}{2} \frac{(N-1) \sin (N h)-N \sin ((N-1) h)}{\cos (h)-1} .
$$

\subsection{Simulation results}

Simulations are performed with $N=10$ observations and an a priori variance $\sigma_{\theta}^{2}=0.5$. On fig. 1 we have plotted the $\mathrm{BCRB}$, the BZB and the proposed bound versus SNR. The advantage of the proposed bound is a better prediction of the SNR threshold in comparison with the BZB $(5 \mathrm{~dB}$ in this case). We can also note that this result has been obtained with approximatively the same computational cost than the BZB.

Moreover, in the transition region between asymptotic and prior areas, we can observe, concerning the tightness, the improvement obtained with the proposed bound.

\section{CONCLUSION}

This paper presents a new unified approach for the obtention of the Bayesian bounds which derive from a covariance inequality principle (the so-called Weiss-Weinstein family). By the way of a "continuously constrained" optimization problem, we obtain the MMSEE MSE, and by judiciously sampling the continuum of constraints, we obtain a method to establish this kind of Bayesian bounds. Moreover, this approach allow us to derive new bounds illustrated by the introduction of a new bound which can be see as a Bayesian version of the Abel bound. 


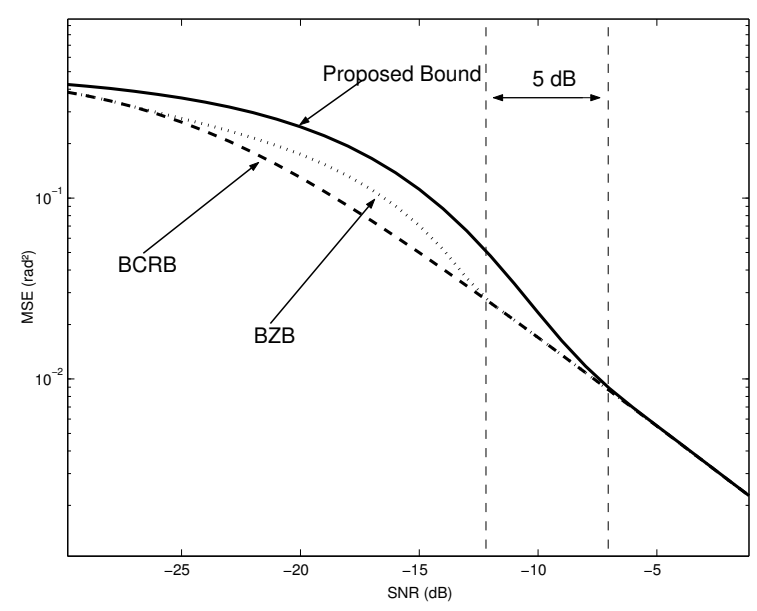

Fig. 1. BCRB, BZB and proposed bound versus SNR

\section{APPENDIX: PROOFS OF THEOREM 2,3 AND 4}

Proof of theorem 2: $\forall f(\mathbf{x}, \theta) \iint_{\Omega} v(\mathbf{x}, \theta) \frac{\partial f(\mathbf{x}, \theta)}{\partial \theta} d \theta d \mathbf{x}=1$ then

$$
\forall f(\mathbf{x}, \theta) \iint_{\Omega} \frac{\partial v(\mathbf{x}, \theta)}{\partial \theta} f(\mathbf{x}, \theta) d \theta d \mathbf{x}=-1,
$$

since

$$
\begin{gathered}
\int_{\Theta} v(\mathbf{x}, \theta) \frac{\partial f(\mathbf{x}, \theta)}{\partial \theta} d \theta= \\
\underbrace{[v(\mathbf{x}, \theta) f(\mathbf{x}, \theta)]_{-\infty}^{+\infty}}_{=0}-\int_{\Theta} \frac{\partial v(\mathbf{x}, \theta)}{\partial \theta} f(\mathbf{x}, \theta) d \theta .
\end{gathered}
$$

The eqn. (41) is for all pdf $f(\mathbf{x}, \theta)$, then, if we put $f(\mathbf{x}, \theta)=\delta\left(\mathbf{x}-\mathbf{x}_{0}, \theta-\theta_{0}\right)$, where $\delta($.$) is the Dirac dis-$ tribution, we have $\forall \mathbf{x}_{0}$ and $\forall \theta_{0}$

$$
\left.\frac{\partial v(\mathbf{x}, \theta)}{\partial \theta}\right|_{\mathbf{x}_{0}, \theta_{0}}=-1 \Longrightarrow v(\mathbf{x}, \theta)=q(\mathbf{x})-\theta
$$

where $q(\mathbf{x})$ is function of $\mathbf{x}$ only.

On the other hand, if we have $v(\mathbf{x}, \theta)=q(\mathbf{x})-\theta$, then

$$
\begin{gathered}
\forall f(\mathbf{x}, \theta) \iint_{\Omega} v(\mathbf{x}, \theta) \frac{\partial f(\mathbf{x}, \theta)}{\partial \theta} d \theta d \mathbf{x} \\
=\iint_{\Omega}(q(\mathbf{x})-\theta) \frac{\partial f(\mathbf{x}, \theta)}{\partial \theta} d \theta d \mathbf{x} \\
=\int_{\Omega} \underbrace{[(q(\mathbf{x})-\theta) f(\mathbf{x}, \theta)]_{-\infty}^{+\infty}}_{=0}+\int_{\Theta} f(\mathbf{x}, \theta) d \theta d \mathbf{x} \\
=1,
\end{gathered}
$$

which complets the proof.

Proof of theorem 3: $\forall f(\mathbf{x}, \theta)$ and $\forall h$

$$
\begin{aligned}
& \iint_{\Omega} v(\mathbf{x}, \theta)(f(\mathbf{x}, \theta+h)-f(\mathbf{x}, \theta)) d \theta d \mathbf{x}=h \\
& \left.\underset{h=0}{\Rightarrow} \iint_{\Theta} v(\mathbf{x}, \theta) \frac{\partial f(\mathbf{x}, \theta+h)}{\partial h}\right|_{h=0} d \theta d \mathbf{x}=1 \\
& \Rightarrow \int_{\Omega} \int_{\Theta} v(\mathbf{x}, \theta) \frac{\partial f(\mathbf{x}, \theta)}{\partial \theta} d \theta d \mathbf{x}=1 \\
& \Longrightarrow v(\mathbf{x}, \theta)=q(\mathbf{x})-\theta \text {, }
\end{aligned}
$$

where the last implication is given by the theorem 2 .

On the other hand, if we have $v(\mathbf{x}, \theta)=q(\mathbf{x})-\theta$, then

$$
\begin{aligned}
& \iint_{\Omega} v(\mathbf{x}, \theta) f(\mathbf{x}, \theta+h) d \theta d \mathbf{x} \\
= & \int_{\Omega} \int_{\Theta}(q(\mathbf{x})-\theta) f(\mathbf{x}, \theta+h) d \theta d \mathbf{x} \\
== & \iint_{\Omega}(q(\mathbf{x})-\varphi+h) f(\mathbf{x}, \varphi) d \varphi d \mathbf{x} \\
= & \int_{\Omega} \int_{\Theta}(q(\mathbf{x})-\varphi) f(\mathbf{x}, \varphi) d \varphi d \mathbf{x}+h,
\end{aligned}
$$

which leads to

$$
\iint_{\Omega}(q(\mathbf{x})-\theta)(f(\mathbf{x}, \theta+h)-f(\mathbf{x}, \theta)) d \theta d \mathbf{x}=h,
$$

which complets the proof.

Proof of theorem 4: $\forall f(\mathbf{x}, \theta), \forall h$ and $\forall s$

$$
\begin{gathered}
\int_{\Omega} \int_{\Theta} v(\mathbf{x}, \theta) w(\mathbf{x}, \theta, h, s) f(\mathbf{x}, \theta) d \theta d \mathbf{x} \\
=h \iint_{\Theta}\left(\frac{f(\mathbf{x}, \theta-h)}{f(\mathbf{x}, \theta)}\right)^{1-s} f(\mathbf{x}, \theta) d \theta d \mathbf{x} \\
\Rightarrow \int_{s=1} \int_{\Omega} v(\mathbf{x}, \theta)\left(\left(\frac{f(\mathbf{x}, \theta+h)}{f(\mathbf{x}, \theta)}\right)-1\right) f(\mathbf{x}, \theta) d \theta d \mathbf{x}=h \\
\Rightarrow \int_{\Omega} \int_{\Theta} v(\mathbf{x}, \theta)(f(\mathbf{x}, \theta+h)-f(\mathbf{x}, \theta)) d \theta d \mathbf{x}=h \\
\Longrightarrow v(\mathbf{x}, \theta)=q(\mathbf{x})-\theta,
\end{gathered}
$$

where the last implication is given by the theorem 3 . 
On the other hand, if we have $v(\mathbf{x}, \theta)=q(\mathbf{x})-\theta$, then

$$
\begin{gathered}
\iint_{\Omega} v(\mathbf{x}, \theta)\left(\frac{f(\mathbf{x}, \theta+h)}{f(\mathbf{x}, \theta)}\right)^{s} f(\mathbf{x}, \theta) d \theta d \mathbf{x} \\
=\iint_{\Omega}(q(\mathbf{x})-\theta)\left(\frac{f(\mathbf{x}, \theta+h)}{f(\mathbf{x}, \theta)}\right)^{s} f(\mathbf{x}, \theta) d \theta d \mathbf{x} \\
=\iint_{\Theta}(q(\mathbf{x})-\varphi+h)\left(\frac{f(\mathbf{x}, \varphi)}{f(\mathbf{x}, \varphi-h)}\right)^{s} f(\mathbf{x}, \varphi-h) d \varphi d \mathbf{x} \\
=\iint_{\Theta}(q(\mathbf{x})-\varphi)\left(\frac{f(\mathbf{x}, \varphi-h)}{f(\mathbf{x}, \varphi)}\right)^{1-s} f(\mathbf{x}, \varphi) d \varphi d \mathbf{x} \\
+h \int_{\Omega} \int_{\Theta}\left(\frac{f(\mathbf{x}, \varphi-h)}{f(\mathbf{x}, \varphi)}\right)^{1-s} f(\mathbf{x}, \varphi) d \varphi d \mathbf{x},
\end{gathered}
$$

which leads to

$$
\begin{aligned}
& \int_{\Omega} \int_{\Theta} v(\mathbf{x}, \theta) w(\mathbf{x}, \theta, h, s) f(\mathbf{x}, \theta) d \theta d \mathbf{x} \\
& =h \int_{\Omega} \int_{\Theta}\left(\frac{f(\mathbf{x}, \theta-h)}{f(\mathbf{x}, \theta)}\right)^{1-s} f(\mathbf{x}, \theta) d \theta d \mathbf{x},
\end{aligned}
$$

which complets the proof.

\section{REFERENCES}

[1] P. Forster and P. Larzabal, "On lower bounds for deterministic parameter estimation," in Proc. IEEE Int. Conf. Acoust., Speech, Signal Processing, (Orlando, Fl), 2002.

[2] F. E. Glave, "A new look at the Barankin lower bound," IEEE Trans. Inform. Theory, vol. 18, pp. 349356, May 1972.

[3] H. L. V. Trees, Detection, Estimation and Modulation Theory, vol. 1. New York: Wiley, 1968.

[4] W. Xu, A. B. Baggeroer, and K. Bell, "A bound on mean-square estimation with background parameter mismatch," IEEE Trans. Inform. Theory, vol. 50, pp. 621-632, Apr. 2004.

[5] W. Xu, A. B. Baggeroer, and C. D. Richmond, "Bayesian bounds for matched-field parameter estimation," IEEE Trans. Signal Processing, vol. 52, pp. 3293-3305, Dec. 2004.

[6] J. Ziv and M. Zakai, "Some lower bounds on signal parameter estimation," IEEE Trans. Inform. Theory, vol. 15, pp. 386-391, May 1969.

[7] E. Weinstein and A. J. Weiss, "Lower bounds on the mean square estimation error," Proceedings of the IEEE, vol. 73, pp. 1433-1434, Sept. 1985.
[8] S. Bellini and G. Tartara, "Bounds on error in signal parameter estimation," IEEE Trans. Commun., vol. 22, pp. 340-342, Mar. 1974.

[9] D. Chazan, M. Zakai, and J. Ziv, "Improved lower bounds on signal parameter estimation," IEEE Trans. Inform. Theory, vol. 21, pp. 90-93, Jan. 1975.

[10] E. Weinstein, "Relations between Belini-Tartara, Chazan-Zakai-Ziv, and Wax-Ziv lower bounds," IEEE Trans. Inform. Theory, vol. 34, pp. 342-343, Mar. 1988.

[11] B. Z. Bobrovsky and M. Zakai, "A lower bound on the estimation error for certain diffusion processes," IEEE Trans. Inform. Theory, vol. 22, pp. 45-52, Jan. 1976.

[12] K. Bell, Performance bounds in parameter estimation with application to bearing estimation. $\mathrm{PhD}$ thesis, George Mason University, Fairfax, VA, 1995.

[13] E. Weinstein and A. J. Weiss, "A general class of lower bounds in parameter estimation," IEEE Trans. Inform. Theory, vol. 34, pp. 338-342, Mar. 1988.

[14] I. Reuven and H. Messer, "A Barankin-type lower bound on the estimation error of a hybrid parameter vector," IEEE Trans. Inform. Theory, vol. 43, pp. 1084-1093, May 1997.

[15] J. S. Abel, "A bound on mean-square-estimate error," IEEE Trans. Inform. Theory, vol. 39, pp. 1675-1680, Sept. 1993. 